\title{
Psoriasis: The Immune System Disease with Unknown Etiology Treated with Local Bath PUVA
}

\author{
Ehsani A, Nourmohammadpour P, Shojaei T and Beigi PK* \\ Misdiagnosis Association, USA
}

*Corresponding author: Dr. Pooya Khan Beigi, Misdiagnosis Association, 244 NW Market Street Seattle, WA, 98107 USA, Tel: 1-206-299 0940; Email: misdiagnosis.association@gmail.com

\section{Research Article \\ Volume 3 Issue 1}

Received Date: November 11, 2018

Published Date: March 29, 2019

DOI: $10.23880 /$ cdoaj-16000144

\section{Abstract}

Psoriasis is a chronic and lifetime disease which relates to the immune system, having unknown etiology. It typically emerges with dermal disorders and sometimes with joint disorders. Psoriasis Palmoplantar is a kind of localized chronic dermatosis in palms of hands and soles of feet; the significance of which is its resistance to treatment and high rate of reoccurrence. Psoriasis Palmoplantar significantly influences the quality of patients' lives due to affecting their everyday life tasks.

Procedure: This is a descriptive analytical study, performed in retrospective method. The target society for this study includes all patients suffering from Psoriasis Palmoplantar, who referred to X-Ray Clinic of Razi Hospital during the years of 2005 to 2009 and went under treatment using Local Bath PUVA. Questionnaires were made subject to the data recorded in medical files of patients.

Results: Medical files of 95 patients, suffering from Psoriasis Palmoplantar, were investigated in this study. The patients, who referred, went under treatment using Local Bath PUVA at Razi Hospital. 49.5\% of the patients were male and 50.5\% were female. Average age of patients was 44.17 years. The youngest patient was 6 years old and the oldest was 81 years old. Most of patients were in 4th and 6th decades in age. Average number of treatment sessions for the studied patients were 42.5 sessions and average collective radiation dose was J/CM2 251.8.

Occurrence rate of side effects in studied patients was $20 \%$. In final assessment, $16.8 \%$ of patients had a very good response to treatments ( $75 \%$ to $100 \%$ recovery of disordered dermal surfaces). $35.8 \%$ of patients had a good response to treatments (50\% to $75 \%)$ and $29.5 \%$ of patients had an average response to treatments (25\% to $50 \%$ ) and $9.5 \%$ of patients had a weak response to treatments (less than $25 \%$ ) and $8.4 \%$ of patients showed complete failure in treatments. In statistical terms, collective radiation dose for patients with very good treatment response (more than 75\%) was significantly higher than other patients. The number of treatment sessions in these individuals was significantly higher than other patients. But among those patients with lack of recovery, low recovery and average and good recovery, there was no significant difference in number of sessions or dose of radiation. There was no significant difference between the 
gender of the patients and their response to the treatment (P: .043). Average age of patients with good recovery response was significantly higher than those with no recovery response (P: .018).

Keywords: Psoriasis; Psoriasis Palmoplantar; Local Bath PUVA; 95 patients; Chronic dermatosis

Abbreviations: PUVA: Psolaren and Ultra Violet radiation spectrum $A$.

\section{Introduction}

\section{Etiology}

Psoriasis is inherited genetically and is a dominant autosomal disease, with variable penetrations rates. The cause for Psoriasis is unknown, but some people are more susceptible to its occurrence.

Studies showed that Psoriasis is a self immune disease, relating to types of B17, B37 and B13 HLA.

\section{Pathogenesis}

Although the pathogenesis of Psoriasis is still unknown, there are clear evidences that Psoriasis is the result of interaction between innate and adaptive immune system in skin and joints. Scientists believe that in Psoriasis, the immune system causes an increase in growth and activity of Mitotic Keratinocytes of basal layer in medium of $\mathrm{T}$ Cells, and decreases immigration duration of Keratinocytes from basal to surface to 3-5 days from 28 days in the patients.

\section{Clinical Exposures}

Psoriasis is a Papulosquamous disease with morphology, distribution, severity and variable courses. Psoriasis lesions are in circular plaques and Popules with certain boundaries, the surface of which is covered with white and silver sticky and dry skins.

\section{Psoriasis Palmoplantar}

Psoriasis occurs on palms of hands and soles of feet in form of sloughing patches which is the result of scratching lesions, where thin silver skin exposes on the place or they expose in form of plaques with unclear limits. It is similar to hyperkeratotic eczema or as simplex; in some cases, they expose as pustulosis.

Psoriasis may occur on the back of hands or feet in form of increasing in thickness and redness of skin; but, it is usually in typical form on palms of hands and soles of feet. There may be a correlation between disease, trauma, and occupational conditions of patients.

\section{Epidemiology}

Pustulosis Palmoplantar has global widespread. Although it is considered rare relative to other types of Psoriasis, no accurate prevalence data is present for it. It sounds that this disease has more prevalence in females than males with a rate of 3:1. Prevalence of the disease is mostly between the ages of 20-60 years old [1-5].

\section{Treatment}

PPP disease is resistant to treatment and its high prevalence is reported in all treatment methods. The first step in treating the disease is advising the patients to quit smoking cigarette. In patients with low tolerance to gluten, gluten free diet positively affects Psoriasis skin lesions. Considering that skin abruption in palms of hands and soles of feet is low and even in acute attacks of disease which skin surface is locally destructed, only special medicines may effectively act. Potent and super potent local steroids are selective medicines and they may be used under plastic cover or under hydrocolloid wound dressing as initial treatment [3].

Other local treatments including Vit D3 analogues such as Calcipotriol Calcipotriene, Tazarotene and Athralin, may prevent early recurrence of the disease in some patients. Long term use of local steroids is usually required for reaching treatment targets. Treatment with Psolaren and Ultra Violet radiation spectrum A (PUVA) is an effective treatment method solely or in combination with systemic retinoid. PUVA Bath or application of PUVA with gel or Photosensitizing creams in case control studies show effective effects. In disabling cases and repeated reoccurrences of PPP, systemic retinoid is the only effective treatment option [6,7].

Photochemotherapy with Psoralen: The experience of treating Psoriasis by Coal-tar and UV and also, Photochemotherapy with Psoralen in treatment of Vitiligo, has suggested that similar treatment could be effective for Psoriasis. Later, studies proved the effectiveness of local treatment and edible one accompanied by PUVA in treating different patterns of 
Psoriasis. PUVA treatment along with local Psoralen may prevent some side effects of which arise in the edible treatment; it is an option in treating patients with localized forms of disease in which local treatment repeats for 2-5 times a week. Dose of UVA gradually increases with regard to the treatment response of patient [8-11].

\section{The Studies Society}

The study society covers all Psoriasis Palmoplantar patients who were referred to the radiation clinic of Razi Hospital for treatment with local bath PUVA between years of 2005 to $2009[12,13]$.

\section{Methodology}

\section{Data Collection Method}

The main objective of this study is observing the patience response to treatment with local bath PUVA, the patients were divided into 5 groups based on the optimization value of affected skin area in patients:

1 . The group with no treatment recovery

2. Improvement in less than $25 \%$ of skin lesions of affected skin lesions

3. Improvement in $25-50 \%$ of skin lesions of affected skin area

4. Improvement in 50-75\% of skin lesions of affected skin area

5. Improvement in $75-100 \%$ of skin lesions of affected skin area

\section{Implementation Method}

Files of all Psoriasis Palmoplantar patients referred to the radiation clinic of Razi Hospital for undergoing treatment with Local Bath PUVA from 2005 to 2009.

\section{Data Analysis Method}

SPSS software (Version 15) was used for data analysis and T-test guideline was used for quantitative variables; Chi-square was used for qualitative variables and Fisher order was also used for required cases. $\mathrm{P}$ was defined significantly in 0.05 .

\section{Results}

In this study, files of 95 patients suffering from Psoriasis Palmoplantar who referred to the radiation clinic of Razi Hospital from 2005 to 2009. From the 95 studied cases, 47 were male (49.5\%) and 48 were female (50.5\%) (Table 1).

\begin{tabular}{|c|c|c|}
\hline Gender of patients & Number & $\mathbf{\%}$ \\
\hline Male & 47 & $49.50 \%$ \\
\hline Female & 48 & $50.50 \%$ \\
\hline Total & 95 & $100 \%$ \\
\hline
\end{tabular}

Table 1: Frequency of studied patients in terms of gender.

Age of patients was investigated at the starting date of treatments. The youngest patient was 6 years old and the oldest was 81 years old. Average age of the patients was 44.17 years. Frequency of the age of patients in terms of age decade was divided into 8 groups; where, the 4th and 6 th decades had the highest group individuals (Table 2).

\begin{tabular}{|c|c|c|}
\hline Age decades of patients & Number & $\mathbf{\%}$ \\
\hline $0-10$ & 2 & 2.1 \\
\hline $10-20$ & 8 & 8.4 \\
\hline $20-30$ & 11 & 11.6 \\
\hline $30-40$ & 20 & 21.1 \\
\hline $40-50$ & 17 & 17.9 \\
\hline $50-60$ & 20 & 21.1 \\
\hline $60-70$ & 13 & 13.7 \\
\hline $70-80$ & 3 & 3.2 \\
\hline $80-90$ & 1 & 1.1 \\
\hline Total & 95 & 100 \\
\hline
\end{tabular}

Table 2: Frequency of the age of studied patients in terms of age decades.

In 95 studied cases, the least number of treatment sessions was 10 sessions and maximum number of which was 120 sessions; in average, 42.5 sessions was with standard deviation of 20.9 treatment sessions. Most of the individuals had at least 30 to 35 treatment sessions. In 95 studied cases, the average collective radiation dose in patients was J/CM2 251.8 with standard deviation of 203.4. Least received collective radiation dose in above study was 15.2 and the highest rate of which was 1209.8 .

57 of the patients continued treatments up to almost complete recovery and completed the treatment subject to orders of attending physicians. 13 patients (13.7\%) stopped treatments because of short-term side effects including irritation, pruritus and erythema subject to orders of attending physicians or they stopped treatments without attending to the orders of attending physicians. 10 patients $(10.5 \%)$ stopped treatments because of observing no treatment improvements; 15 patients stopped treatments with no reason mentioned in files and against the orders of attending physicians (Table 3). 


\begin{tabular}{|c|c|c|}
\hline Cause of stopping treatments & Number & $\%$ \\
\hline Complete response or maximum response to treatment & 57 & $60 \%$ \\
\hline Short-term side effects & 13 & $13.70 \%$ \\
\hline Lack of receiving acceptable treatment response & 10 & $10.50 \%$ \\
\hline Low compliance of patients & 15 & $15.80 \%$ \\
\hline Total & 95 & $100 \%$ \\
\hline
\end{tabular}

Table 3: Frequency of the cause of stopping treatments in studied patients

Values of collective radiation dose in patients based on the treatment recovery value were as follows (Table 4):

\begin{tabular}{|c|c|c|c|c|c|}
\hline $\begin{array}{c}\text { Collective radiation dose } \\
\text { Value of Recovery }\end{array}$ & Number & Average & Standard Deviation & Minimum & Maximum \\
\hline 0 & 8 & 152.775 & 98.7633 & 34.6 & 312 \\
\hline Less than 25\% & 9 & 111.556 & 82.0589 & 15.2 & 254.6 \\
\hline $25 \%-50 \%$ & 28 & 253.543 & 209.9667 & 32 & 1060 \\
\hline $50 \%-75 \%$ & 34 & 233.712 & 121.8149 & 60.6 & 481.6 \\
\hline $75 \%-100 \%$ & 16 & 416.05 & 304.2764 & 120.2 & 1209.8 \\
\hline Total & 95 & 251.878 & 203.4081 & 15.2 & 1209.8 \\
\hline
\end{tabular}

Table 4: Value of collective radiation dose in studied patients regarding their treatment response

Among 95 studied patients, 19 patients (20\%) suffered from short-term side effects including irritation, pruritus and erythema in skin and 76 patients (80\%) experienced no side effect (Table 5).

\begin{tabular}{|c|c|c|}
\hline Side effect & Number & $\mathbf{\%}$ \\
\hline Yes & 19 & $20 \%$ \\
\hline No & 76 & $80 \%$ \\
\hline Total & 95 & $100 \%$ \\
\hline
\end{tabular}

Table 5: Frequency of short-term side effects in patients undergoing treatment with Local Bath PUVA

Frequency of short-term side effects in patients was investigated based on gender and there was no significant difference between genders in terms of side effects ( $P$ : 0.473) (Table 6).

\begin{tabular}{|c|c|c|c|}
\hline Short-term side effects & Male & Female & Total \\
\hline Yes & 8 & 11 & 19 \\
\hline No & 39 & 37 & 76 \\
\hline Total & 47 & 48 & 95 \\
\hline
\end{tabular}

Table 6: Frequency of short-term side effects based on gender of patients

Frequency of short-term side effects in patients was investigated in terms of age; there was no significant difference in occurrence of side effects in terms of age. (P: 0.814) (Table 7).

\begin{tabular}{|c|c|c|c|}
\hline $\begin{array}{c}\text { Short-term side } \\
\text { effects }\end{array}$ & Number & $\begin{array}{c}\text { Age } \\
\text { average }\end{array}$ & $\begin{array}{c}\text { Standard } \\
\text { deviation }\end{array}$ \\
\hline Yes & 19 & 44.11 & 18.14 \\
\hline No & 76 & 44.18 & 16.01 \\
\hline
\end{tabular}

Table 7: Average age of patients in experiencing side effects

Based on treatment recovery, patients were divided into 5 groups including:

1. The group with no treatment recovery

2. Treatment recovery was assessed weak for patients with less than $25 \%$ in lesions recovery

3. Treatment recovery was assessed average for patients with less than $25 \%-50 \%$ in lesions recovery

4. Treatment recovery was assessed good for patients with less than $50 \%-75 \%$ in lesions recovery

5. Treatment recovery was assessed very good for patients less than $75 \%-100 \%$ in lesions recovery

In 95 studied patients, $16(16.8 \%)$ patients had a very good recovery; 34 (35.8\%) patients had a good recovery; $28(29.5 \%)$ patients had an average recovery; 9 (9.5\%) patients had a weak recovery. 8 patients experienced no recovery (Table 8 ). 


\begin{tabular}{|c|c|c|}
\hline Value of Recovery & Frequency & \% \\
\hline 0 & 8 & 8.4 \\
\hline $0-25 \%$ & 9 & 9.5 \\
\hline $25-50 \%$ & 28 & 29.5 \\
\hline $50-75 \%$ & 34 & 35.8 \\
\hline $75-100 \%$ & 16 & 16.8 \\
\hline Total & 95 & 100 \\
\hline
\end{tabular}

Table 8: Frequency of patients subject to value of recovery after treatment with Local Bath PUVA.

In comparison of the rate of response to treatment and using Independent Sample T-test (P: .063) (Table 9). gender of patients, no significant difference was observed

\begin{tabular}{|c|c|c|c|c|c|c|c|}
\hline \multicolumn{2}{|c|}{ Value of recovery } & no recovery & Low & Average & Good & Very good & Total \\
\hline \multirow{2}{*}{ Male } & Number & 5 & 5 & 11 & 15 & 11 & 47 \\
\cline { 2 - 9 } & $\%$ & 10.6 & 10.6 & 23.4 & 31.9 & 23.4 & 100 \\
\hline \multirow{2}{*}{ Female } & Number & 3 & 4 & 17 & 19 & 5 & 48 \\
\cline { 2 - 9 } & $\%$ & 6.2 & 8.3 & 35.4 & 39.5 & 10.4 & 100 \\
\hline \multicolumn{2}{|c|}{ Total } & 8 & 9 & 28 & 34 & 16 & 95 \\
\hline
\end{tabular}

Table 9: Frequency of groups with different recovery values in both genders.

There was significant difference between average age and value of recovery of intergroup patients with very good response to treatment $(75-100 \%)$ and the group with no recovery response (P: .018); i.e. average age of patients with very good recovery response was significantly higher than the group with no recovery response; this shows direct correlation of the recovery and age. Although, average age of patients in all groups increased with response to treatment, this difference was not significant in all groups (Table 10).

\begin{tabular}{|c|c|c|c|c|}
\hline $\begin{array}{c}\text { Age } \\
\text { Recovery } \\
\text { response }\end{array}$ & Average & $\begin{array}{c}\text { Standard } \\
\text { Deviation }\end{array}$ & Maximum & Minimum \\
\hline 0 & 33 & 10.7 & 50 & 18 \\
\hline $0-25 \%$ & 41.7 & 21.5 & 81 & 15 \\
\hline $25-50 \%$ & 42.3 & 15.5 & 62 & 6 \\
\hline $50-75 \%$ & 46.2 & 15.8 & 76 & 11 \\
\hline $75-100 \%$ & 49.8 & 16.3 & 75 & 29 \\
\hline
\end{tabular}

Table 10: Age average in groups with different recovery responses.

In comparison of collective radiation dose between groups, there was a significant difference between the dose for patients in the very good recovery group and rest of patients in the rest of groups. $(\mathrm{P}<0.05)$; value of $\mathrm{P}$ in comparison of collective radiation dose between groups with different treatment recovery were as follows (Table 11):

\begin{tabular}{|c|c|c|}
\hline Groups in Comparison & Compared Group & $\mathbf{P}$ \\
\hline \multirow{4}{*}{0} & $0-25 \%$ & 0.991 \\
\cline { 2 - 3 } & $25-50 \%$ & 0.67 \\
\cline { 2 - 3 } & $50-75 \%$ & 0.809 \\
\cline { 2 - 3 } & $75-100 \%$ & 0.015 \\
\hline \multirow{5}{*}{$0-25 \%$} & 0 & 0.991 \\
\cline { 2 - 3 } & $25-50 \%$ & 0.29 \\
\cline { 2 - 3 } & $50-75 \%$ & 0.421 \\
\hline \multirow{5}{*}{$25-50 \%$} & $75-100 \%$ & 0.002 \\
\cline { 2 - 3 } & 0 & 0.67 \\
\cline { 2 - 3 } & $25-50 \%$ & 0.29 \\
\cline { 2 - 3 } & $50-75 \%$ & 0.994 \\
\hline \multirow{5}{*}{$50-75 \%$} & $75-100 \%$ & 0.054 \\
\cline { 2 - 3 } & 0 & 0.809 \\
\cline { 2 - 3 } & $25-50 \%$ & 0.421 \\
\cline { 2 - 3 } & $50-75 \%$ & 0.994 \\
\hline \multirow{5}{*}{$75-100 \%$} & $75-100 \%$ & 0.016 \\
\cline { 2 - 3 } & 0 & 0.015 \\
\cline { 2 - 3 } & $25-50 \%$ & 0.002 \\
\cline { 2 - 3 } & $50-75 \%$ & 0.054 \\
\hline \multirow{5}{*}{} & $75-100 \%$ & 0.016 \\
\hline
\end{tabular}

Table 11: P Value in comparison of collective radiation dose in patients with different recovery values.

Also, in comparison of number of treatment sessions, average of treatment sessions in a group of patients with very good recovery response was significantly higher than patients in the other groups $(\mathrm{P}<0.05) ; \mathrm{P}$ value in 
comparison of number of treatment sessions for groups of patients with different recovery response is shows as follows (Table 12).

\begin{tabular}{|c|c|c|}
\hline Groups in Comparison & Compared Group & $\mathbf{P}$ \\
\hline \multirow{4}{*}{0} & $0-25 \%$ & 0.977 \\
\hline & $25-50 \%$ & 0.601 \\
\hline & $50-75 \%$ & 0.608 \\
\hline & $75-100 \%$ & 0.002 \\
\hline \multirow{4}{*}{$0-25 \%$} & 0 & 0.977 \\
\hline & $25-50 \%$ & 0.17 \\
\hline & $50-75 \%$ & 0.467 \\
\hline & $75-100 \%$ & 0 \\
\hline \multirow{4}{*}{$25-50 \%$} & 0 & 0.601 \\
\hline & $25-50 \%$ & 0.17 \\
\hline & $50-75 \%$ & 1 \\
\hline & $75-100 \%$ & 0.007 \\
\hline \multirow{4}{*}{$50-75 \%$} & 0 & 0.608 \\
\hline & $25-50 \%$ & 0.167 \\
\hline & $50-75 \%$ & 1 \\
\hline & $75-100 \%$ & 0.004 \\
\hline \multirow{4}{*}{$75-100 \%$} & 0 & 0 \\
\hline & $25-50 \%$ & 0.007 \\
\hline & $50-75 \%$ & 0.004 \\
\hline & $75-100 \%$ & 0.016 \\
\hline
\end{tabular}

Table 12: P Value in comparison of number of treatment sessions for patients with different recovery values.

\section{Discussion and Conclusion}

From 95 studied patients, 47 were male $(49.5 \%)$ and 48 were female $(50.5 \%)$; this shows similar prevalence in both genders. In a Japanese study, the scientist performed an epidemiological study on 28628 patients with Psoriasis. The data suggested that $65.5 \%$ of the individuals were male and $34.2 \%$ were female. The data indicated a dominant prevalence of this disease in Japanese males. In addition, French scientists performed a study on 99 patients with Psoriasis in 2004. They concluded that the prevalence ratio of the disease in males relative to females was 0.77 [14-16].

In our study, average collective radiation dose in treating patients was 251.8. This average in female patients was 225.2 and in male patients was 279.0 which shows no significant difference (P: 0.133). In addition, there was no significant difference between age and collective radiation dose (P: 0.144).
In 95 studied patients, 34 (35.8\%) patients had a good recovery (50\% to $75 \%) .28(29.5 \%)$ patients had average recovery (25\% to $50 \%)$ and $16(16.8 \%)$ patients had very good recovery (higher than $75 \%)$ and $9(9.5 \%)$ patients had weak recovery (less than $25 \%$ ) and $8(8.4 \%)$ patients showed complete failure in treatments; i.e. highest rate of effectiveness of treatment with Local Bath PUVA in our study was about $50-75 \%$.

This study showed significant difference between response to treatment and collective radiation dose; in a way that value of collective radiation dose in patients with very good treatment response (higher than 75\%) was significantly higher than patients with no recovery response, low recovery response, and good recovery response $(50-75 \%)$.

This shows indicates a direct correlation of collective radiation dose and recovery response. There was a similar significant correlation in terms of number of treatment sessions between patients with very good recovery response and other patients. Also, average age of patients with very good recovery response was significantly higher than average age of patients with no recovery response. This may indicate a direct correlation between age and recovery response in patients. However, no other similar study has any findings to support this conclusion.

Major goal of our study deals with investigating effectiveness of Local Bath PUVA on patients suffering from Psoriasis Palmoplantar referred to radiation clinic of Razi Hospital from 2005 to 2009. Our study data suggests that $52.6 \%$ of the patients had a good and very good recovery response; $29.5 \%$ had an average recovery response, and only $8.4 \%$ of patients faced failure in treatment [17-19].

\section{Conclusion and Suggestion}

As mentioned above in our study, collective radiation dose and number of treatment sessions in patients shows a very good recovery response to treatment with Local Bath PUVA indicating that it was significantly higher than other patients; this may show that treatments up to high collective radiation dose to reach recovery response may be very effective; it is required to inform patients and justify it to them before starting treatments and on long duration of this treatment method and achievement of results in long term toward higher compliance of patients with treatment procedures. 
In our study, average age of patients with very good response to treatments was higher than patients with no recovery response, which possibly shows that lower age results in higher treatment failures.

In our study, more than half of patients experienced good and very good recovery responses; failure was only reported for $8 \%$ of patients, and the rest of patients showed weak to average recovery responses. Occurrence of side effects in this study was estimated to be $20 \%$. These results suggest Local Bath PUVA is an effective method in treating Psoriasis Palmoplantar.

\section{References}

1. Langley RG, Krueger CG, Griffiths CE (2005) Psoriasis: epidemiology, clinical features and quality of life. Ann Rheum Dis 64(2): 18-23.

2. Vallat VP, Gilleaudeau P, Battat L, Wolfe J, Nabeya R (1994) PUVA bath therapy strongly suppress immunological and epidermal activation in psoriasis: a possible cellular basis for remittive therapy. Journal of Experimental Medicine 180(1): 283-296.

3. Elder JT, Nair RP, Henseler T, Jenisch S, Stuart P, et al. (2001) The genetics of psoriasis 2001: the odyssey continues. Arch Dermatol 137(11): 1447-1454.

4. Rook's text book of Dermatology. 9th (Edn.), In: Griffiths C, Barker J (Eds.), 20 (Chapter), John Wiley \& Sons, Inc., pp: 15, 29-31.

5. Fitzpatrick 's Dermatology in general medicine. In: Ulrich M Roweitz (Ed), Chapter 21, pp: 215-217.

6. Wahab MA, Amin MN, Khan MAL, Hasan MS (2006) Bath PUVA in treatment of palmoplantar psoriasis, Journal of BANGLADESH, College of Physicians and Surgeons 24(1): 14-18.

7. Legat Franz J, Hofer A, Quehenberger F, Kahofer P, Kerl H, et al. (2004) Reduction of treatment frequency and UVA dose does not substantially compromise the antipsoriatic effect of oral psoralen-UVA. JAAD 51(5): 746-754.

8. Behrens S, von Kobyletzki G, Gruss C, Reuther T, Altmeyer P, et al. (1999) PUVA-bath photochemothrapy (PUVA-soak therapy) of recalcitrant dermatoses of the palms and soles. Photodermatol Photoimmunol Photomed 15(2): 4751.

9. Seçkin D, Yazıcı Z, Ergun T (2009) Efficacy of bath PUVA treatment in palmo plantar psoriasis. Turkderm 43(1): 25-28.

10. Adişen E, Tekin O, Gülekon A, Gürer MA (2009) A retrospective analysis of treatment responses of palmoplantar psoriasis in 114 patients. J Eur Acad Dermatol Venereol 23(7): 814-819.

11. Thomas habif (2015) Clinical dermatology. $6^{\text {th }}$ (Edn).

12. Farber EM, Nall ML (1974) The Natural History of Psoriasis in 5,600 Patients. Dermatologica 148(1): 118.

13. Yip SY (1984) The prevalence of psoriasis in mongoloid race. J Am Acad Dermatol 10(6): 965-968.

14. Augey F, Renaudier P, Nicolas JF (2006) Generalized pustular psoriasis (Zumbusch): A French epidemiological survey. Eur J Dermatol 16(6): 669673.

15. Kimberling W, Dobson RL (1973) The Inheritance of Psoriasis. J Invest Dermatol 60(6): 538-540.

16. Behnam SM, Behnam SE (2005) Smoking \& psoriasis. Skin Med 4(3): 174-176.

17. Murase JE, Chan KK, Garite TJ, Cooper DM, Weinstein GD (2005) Hormonal effect on psoriasis in pregnancy and post partum. Arch Dermatol 141(5): 601-606.

18. Engin B, Oguz O (2005) Evaluation of time-dependent response to psoralen plus UVA (PUVA) treatment with topical 8-methoxypsoralen (8-MOP) gel in palmoplantar dermatoses. Int J Dermatol 44(4): 337339.

19. Delrosso G, Bornacina C, Farinelli P, Bellinzona F, Leigheb G, et al. (2008) Bath PUVA and psoriasis: is a milder treatment a worse treatment? Dermatology 216(3): 191-193. 